\title{
Creencias de los profesores de preescolar y primaria sobre ciencia, tecnología y sociedad, en el contexto de una institución rural
}

\author{
Diana María Ruiz Medina* \\ Leonardo Fabio Martínez Pérez ${ }^{* *}$ \\ Diana Lineth Parga Lozano*** \\ Artículo recibido: 14-04-2009 y aprobado: 12-08-2009 \\ Primary teacher's beliefs about science \\ and technology in the context of a rural institution
}

Resumen:En este artículo se presenta una investigación que busca caracterizar las creencias que tienen algunos profesores de preescolar y primaria sobre ciencia y tecnología en un contexto rural. La metodología usada fue cualitativa, en función de comprender e interpretar la realidad del escenario y participantes observados. Para la recolección de la información se utilizó la entrevista semi-estructurada y la observación participante en un periodo de un año y medio. Algunas de las conclusiones establecidas en el estudio muestran que los profesores creen que la ciencia y la tecnología obedecen a un método, es decir, a una secuencia de pasos rígida y preestablecida imposible de ser apropiada por parte de los estudiantes de las escuelas rurales, así mismo, consideran que el objetivo de la ciencia y la tecnología es su uso en la sociedad, en oposición a la concepción de ciencia y tecnología como disciplinas epistemológicamente autónomas con interrelación y simetría de interdependencia entre ellas.

Palabras clave: Creencias de profesores, relaciones CTS y educación rural.
Abstract: This paper presents a research study aimed at characterizing some preschooler and primary teachers' beliefs about science and technology in a rural context. The methodology used was qualitative for a comprehension and interpretation of the participants and the setting. In order to collect the information two instruments were used: the semi-structured interview addressed to preschool and primary teachers and the participant observation over a period of eighteen months. Some of the conclusions drawn by the researchers show that rural teachers state that science and technology follow a methodology which does not allow its application in rural schools, and they also believe that the goal of science and technology is their application in society.

Key words: Teachers beliefs, CTS relations and rural education.

* Licenciada en psicopedagogía. Universidad Pedagógica Nacional. Correo electrónico: diana12024@gmail.com

** Profesor Departamento de Química. Universidad Pedagógica Nacional. Grupo: ALTERNACIENCIAS. Candidato a Doctor en Educación en Ciencias, Universidade Estadual Paulista "Júlio de Mesquita Filho", Bauru, SP, Brasil. Correo electrónico: lemartinez@pedagogica.edu.co

*** Profesora Departamento de Química. Universidad Pedagógica Nacional. Grupo: ALTERNACIENCIAS. Correo electrónico: dparga@pedagogica.edu.co 


\section{Introducción}

Aunque en sus inicios la mayoría de investigaciones didácticas se ocuparon de caracterizar las actitudes y creencias sobre ciencia, tecnología y sociedad (CTS) de los estudiantes (Acevedo et al., 2002a); en los últimos años, la atención se ha orientado hacia los profesores. En efecto, como han mostrado algunas de las primeras investigaciones, el éxito de la puesta en práctica de los programas CTS depende en gran medida de los profesores, y es por eso que se ha desarrollado un interés por conocer sus creencias respecto de las interacciones CTS en su ambiente escolarizado (Acevedo, Vásquez y Manassero, 2002).

Existe una gran variedad de trabajos de investigación, en el ámbito internacional, relacionados con el análisis de las creencias de profesores acerca de las interacciones CTS. En este sentido, se destaca como antecedente para este estudio, el "Proyecto de Investigación Iberoamericano en evaluación de actitudes relacionadas con la ciencia, la tecnología y la sociedad" realizado por Vásquez y Mannasero (2005), el cual tuvo como perspectiva de trabajo la evaluación de creencias y actitudes de estudiantes y profesores sobre las dimensiones CTS y, a partir de esta evaluación, la articulación de propuestas educativas que mejoren el aprendizaje de los estudiantes y lo que enseñan los profesores en el aula.

Así mismo, se destaca el estudio realizado por Acevedo (2000) sobre las actitudes y creencias sobre CTS del profesorado de secundaria, en ese estudio se compararon las actitudes y creencias sobre CTS del profesorado aplicando el instrumento metodológico COCTS
(Cuestionario de Opiniones y Creencias de Ciencia, Tecnología y Sociedad). Como resultado, los investigadores identificaron creencias de corte empírico inductivo en los profesores observados y diagnosticaron prácticas pedagógicas descontextualizadas y poco pensadas para las necesidades actuales.

En Colombia, algunas universidades están abordando los estudios sobre CTS como parte fundamental de los procesos académicos y sociales. La Universidad del Valle adelanta el "Programa de formación continuada en ciencia, tecnología y sociedad" para docentes de escuelas y colegios de educación básica del municipio Santiago de Cali. También desarrolla una línea transversal de proyectos de aula en los campos de la enseñanza de las ciencias, la educación en tecnología y la educación ambiental.

Por su parte, la Universidad Javeriana junto con la Asociación Colombiana para el Avance de la Ciencia (ACAC) lideran el foro nacional "Presente y Futuro de la Ciencia en Colombia”, motivando a estudiantes en todos los niveles de formación y docentes de todas las áreas, a la reflexión y sistematización de experiencias en torno a la educación científica y tecnológica con responsabilidad social.

En otro escenario, la Universidad del Cauca desarrolla una "Red de Aprendizaje de las Ciencias con Enfoque CTS + I en la Educación Media”, destacándose los trabajos de Corchuelo y Catebiel (2005) sobre la estructuración de orientaciones curriculares con enfoque CTS para la educación media a partir del abordaje de problemas socialmente relevantes.

En la Universidad Pedagógica Nacional se encuentra el grupo de investiga- 
ción "Alternativas para la enseñanza de las ciencias" (ALTERNACIENCIAS) que cuenta con una línea de investigación sobre enseñanza de las ciencias en contextos de ciencia, tecnología, sociedad y ambiente (CTSA) (Parga, 2007). Este grupo ha desarrollado trabajos de investigación sobre estrategias pedagógico-didácticas con enfoque CTSA (Martínez y Rojas, 2006; Martínez, Peña y Villamil, 2007) que involucran, entre otros aspectos, el desarrollo de actitudes favorables hacia la ciencias y el diseño de actividades didácticas para fomentar la alfabetización científica. Este artículo hace parte de la dinámica de este grupo de investigación, y se preocupa específicamente del estudio de las creencias de profesores de preescolar y primaria acerca de las relaciones CTS en el contexto rural.

Como antecedentes, de los estudios CTSA del grupo ALTERNACIENCIAS, encontramos los trabajos de Guerrero y Cabrera (2002; 2004) que apuntan a la construcción de estrategias de enseñanza de la química desde una perspectiva interdisciplinaria, a partir del estudio de problemas socio-ambientales como la emisión de $\mathrm{CO}_{2(\mathrm{~g})}$ y la discusión del impacto ambiental de los fluorocarbonos (Guerrero, 2000). También fue un referente el análisis epistemológico de Gallego, Torres y Aristizábal (2004) sobre el abordaje de la relaciones CTS en los estándares curriculares de ciencias naturales promovido por el Ministerio de Educación Nacional.

Hay que mencionar también, como trabajo relacionado directamente con las relaciones CTS en el contexto rural, a Martínez y Reyes (2006) quienes concluyeron que las relaciones CTS no son tenidas en cuenta en la enseñanza de las ciencias, por los maestros, y tampoco son comprendidas, por los estudiantes de educación media, en el caso concreto del Colegio Departamental Tapias, de la vereda Cerezos, municipio Chipaque.

En cuanto a las investigaciones sobre CTS y formación de profesores de educación infantil, hay que destacar el trabajo de (Arana et al., 2006) quienes identificaron las imágenes que se dan, sobre ciencia y tecnología, en un grupo de profesores y estudiantes de los programas curriculares de Educación Infantil de la Universidad Pedagógica Nacional y Pedagogía Infantil de la Universidad Libre de Colombia. Dentro de las conclusiones de este estudio hay que señalar como el grupo presenta una percepción de la ciencia descontextualizada y alejada de la sociedad, además de una imagen de la tecnología asociada a aplicaciones del conocimiento científico y una reducción de la actividad científica y tecnológica a aspectos económicos que se imponen en tanto que fomentan la competitividad y productividad de bienes materiales.

Teniendo en cuenta que, luego de una revisión de la literatura, se reportan pocos trabajos que estudien las creencias que tienen los profesores de preescolar y primaria sobre las relaciones CTS en escenarios rurales colombianos, esta investigación tuvo dificultades para identificar y analizar las creencias que tienen los profesores de preescolar y primaria sobre CTS en la institución educativa rural Policarpa Salavarrieta, ubicada en el Municipio de Facatativa (Colombia). Este problema permitió plantear la siguiente pregunta orientadora: ¿Cuáles son las creencias que tienen profesores de preescolar y básica primaria, frente 
a las relaciones Ciencia, Tecnología y Sociedad en una institución rural? Como objetivo general se propuso diagnosticar y caracterizar estas creencias, con la perspectiva de vislumbrar procesos de formación del profesor de ciencias en el contexto rural.

Este estudio es importante si se tiene en cuenta que hay un pesimismo general en los profesores colombianos, no solo en la imagen que tienen acerca de la ciencia y la tecnología, sino frente a las posibilidades y capacidades colectivas de emprender cambios a partir de avances científicos y tecnológicos, el 87\% considera la ciencia como parte exclusiva de la globalización, percibiéndola como agente deshumanizador y exclusivo de ciertos contextos sociales (Colciencias, 2005, p. 58).

El diagnóstico de COLCIENCIAS reporta que solo entre $35 \%$ y el $40 \%$ de los docentes participantes del estudio, considera que hay una preocupación real de parte de las instituciones educativas por la experimentación, la utilización de bibliotecas, la actividad investigativa, la divulgación de adelantos científicos y la creación de estrategias educativas. Con la lectura del estudio es posible evidenciar la nula participación de profesores rurales en las reflexiones sobre ciencia y tecnología, teniendo otra razón para indagar acerca de sus creencias sobre CTS.

El fundamento teórico que sigue este trabajo, para efectos de realizar los análisis respectivos, comprende dos aspectos directamente relacionados con el problema planteado. El primero, se refiere al concepto de creencia del profesor y el segundo, abarca algunas consideraciones sobre la educación en CTS y el papel de profesor en este enfoque.

\section{Creencias de los profesores}

Los investigadores de este trabajo compartimos el concepto de creencia planteado por Reyes, Salcedo y Perafán (1999, p. 8) quienes lo definen como algo que se refiere "a todo aquello que en una sociedad es considerado por los sujetos como conocimiento sin detenernos a pensar en su validez". Esta perspectiva nos permite realizar nuestros análisis sin descalificar el pensamiento de los profesores con las determinadas perspectivas teóricas que asumamos.

Siguiendo a los autores citados, la creencia es una construcción humana que nos permite entender el mundo, y de esta manera, guía nuestras acciones y engloba nuestra visión del mismo, desde la cual comprendemos lo que hacemos y lo que hacen todos los sujetos con los que nos relacionamos. De acuerdo con esta comprensión, un conocimiento puede ser una creencia que ha sido construida en determinado contexto y que es validada por éste.

La creencia, desde esta perspectiva, se constituye histórica y culturalmente de las diversas interacciones, vivencias físicas, intelectuales y motivacionales que experimentan los individuos en sus historias de vida particulares. De acuerdo con Perafán (2004, p. 33) "las creencias son construcciones que se han realizado en el proceso de formación como sujetos que permiten entender el mundo, pueden ser consideradas como generadoras de acciones especificas". Así, el sistema de creencias de un profesor es de tal complejidad que aunque no sea consciente de ellas, juega un papel integral en la generación de la acción. 
Las creencias están animadas, según Perafán, en la cultura personal del maestro, en la cultura de los grupos sociales a los cuales pertenece, y en la cultura amplia de la sociedad. En este contexto, las creencias que constituyen las acciones del profesor son un tipo de conocimiento susceptible de ser elaborado a partir de la reflexión, en y sobre la acción, para dar paso a una reivindicación del ejercicio y rol docente como investigador y como intelectual (p. 34). Las creencias juegan un papel importante en la elección de tareas y en la definición de elementos cognitivos para la interpretación, la planificación y la toma de decisiones.

Las acciones y las decisiones de clase de los profesores están relacionadas con sus creencias, ya que los profesores reaccionan menos ante la realidad objetiva que ante las percepciones de esa realidad. Así, el modo en que conciben lo que es cierto está en función de lo que creen que es cierto.

\section{Educación CTS y papel del profesor de ciencias}

La orientación educativa CTS es un campo de estudio e investigación, al tiempo que una propuesta educativa innovadora de carácter general. Desde la primera perspectiva, como campo de estudio, trata de comprender mejor la ciencia y la tecnología en su contexto social, abordando las relaciones mutuas entre los desarrollos científicos y tecnológicos y los procesos de orden social y cultural.

El segundo punto de vista de la CTS, es decir como propuesta educativa, constituye un nuevo planteamiento radical del currículo en todos los niveles de enseñanza, con la principal finalidad de dar una formación en conocimientos y especialmente en valores que favorezcan la participación ciudadana responsable y democrática en la evaluación y control de las implicaciones sociales de la ciencia y la tecnología, en este sentido Acevedo et al (2002b) propone que:

"Los programas de formación del profesorado tienen que prepararse apoyándose expresamente en una cultura científica contextualizada; esto es, no cerrada en sí misma sino abierta a otros saberes como la historia, filosofía y sociología de la ciencia, que constituyen buena parte de la base CTS".

El Movimiento CTS intenta aportar soluciones a diversos problemas de enseñanza y aprendizaje para las ciencias, ya que permite ir mas allá de los contenidos programáticos con su inquietud por los problemas sociales relacionados con lo científico y lo tecnológico, fomentando así, una conciencia social de las realidades que subyacen en diversos contextos.

Adicionalmente, pensar en educación CTS, desde los ambientes de enseñanza preescolar y primaria, implica el fortalecimiento profesional de docentes en ejercicio y profesores en formación permanente, es decir, enriquece los saberes pedagógico-didácticos básicos, que en muchos ambientes, rurales o urbanos, se limitan a la transmisión, mecanización y trabajo manual con poco sentido formativo.

Hay que resaltar como desde la institucionalización del CTS, éste se ha ocupado de estudios dedicados, generalmente, a la formación secundaria y universitaria, y que la mayoría de investigaciones han estado enfocadas en los ambientes urbanos. Sin embargo, la 
puesta en marcha de la perspectiva CTS es pertinente para cualquier contexto y nivel de formación, por tanto, en este trabajo se considera indispensable que el profesor de preescolar y primaria que trabaja en un contexto rural, estudie y discuta los aspectos centrales de este tipo de educación y que sea considerado como un sujeto dinámico y socializador, capaz de crear un ambiente de inquietud y participación al interior del aula.

El profesor debe asumir con responsabilidad y profesionalismo una transformación curricular que motive la investigación, consulta, gestión y diseño de propuestas, que no solo modifiquen las débiles estrategias de enseñanza aprendizaje, sino que también permitan recobrar el estatus de docente intelectual y propositivo, trascendiendo su acción pedagógica y didáctica, al romper las fronteras del aula y arriesgándose a involucrar a sus estudiantes en la democracia, con un amplio espíritu de conciencia política y social. En palabras de Corchuelo y Catebiel (2005, p.7) diríamos lo siguiente:

"Resulta claro que para cumplir los objetivos, en las aulas de clases de la educación se deben generar procesos en los cuales los estudiantes puedan relacionar los aspectos teóricos con la actividad investigativa y su contexto social. Es decir, se requiere de la transformación del aula de clases en un espacio que promueva la participación ciudadana en función de la construcción de alternativas de solución a problemas sociales relevantes del entorno. Entonces, se considera imprescindible construir una propuesta alternativa que supere las limitaciones de los desarrollos curriculares orientados a la transmisión de información, y tenga en cuenta el potencial y las características particulares de los estudiantes, de los docentes y de las instituciones".

Teniendo en cuenta los conceptos presentados, se describe a continuación la metodología y los resultados obtenidos en el trabajo.

\section{Consideraciones Metodológicas}

La metodología utilizada fue de corte cualitativo, y se caracteriza por ser exploratoria e interpretativa, asumiendo que el compromiso del investigador es el significado social. Con ello, la relación entre sujeto e investigador es cercana y comprensiva, por lo cual, los datos son textuales y detallados diferenciándose así del enfoque cuantitativo, que busca la objetividad acrítica y neutra que está sujeta a los cánones de la medición.

Siguiendo a Álvarez-Gayout (2004) la investigación cualitativa busca explicar y comprender las interacciones y significados que tiene un individuo o un colectivo en particular desde la subjetividad; lo que se espera al final es una descripción, una comprensión experiencial y múltiples realidades.

La investigación se realizó con 6 profesores de preescolar y primaria', de las sedes Santa Sofía y San Rafael de la Institución Educativa Policarpa Salavarrieta, ubicada en el Municipio de Facatativa, departamento de Cundinamarca. Esta institución, fundada hace más de 30 años, como escuela rural, cuenta con todos los niveles de la

1 De acuerdo con la ley 115 de 1994 (ley general de educación) el nivel preescolar corresponde a los primeros tres grados de educación escolarizada, siendo uno obligatorio, mientras que el nivel de educación básica primaria estaría conformado por 5 grados obligatorios posteriores al nivel preescolar. 
educación básica primaria, secundaria y media vocacional.

La institución tiene un solo profesor por cada grado desde preescolar hasta primaria, dicho profesor imparte todas las áreas al curso respectivo, en bachillerato, hay rotación docente por áreas específicas. De acuerdo a la misión y visión propuestas en el PEI, se desarrollan proyectos productivos de agricultura, cría de conejos, granja y huerta escolar.

La investigación trabaja con profesores que imparten el área de ciencias naturales en la educación básica y en preescolar junto con las otras materias del currículo, en su mayoría son Licenciados en educación y preescolar, y algunos son normalistas o técnicos agropecuarios.

Fueron entrevistados un total de 6 profesores y se realizaron observaciones participantes a 4 de ellos durante un año y medio. La entrevista usada fue semiestructurada porque ofrecía mayor libertad tanto al entrevistador como al entrevistado, y utilizó preguntas abiertas que fueron respondidas naturalmente, tuvo como característica principal la ausencia de una estandarización formal (Ander-Egg, 1978). La observación participante realizada consistió en una vinculación de la autora principal de este trabajo con la situación que se observó, en este proceso adquirió responsabilidades en las actividades del grupo sin convertirse en un miembro del mismo (Del Rincón et al., 1995).

\section{Resultados y análisis: creencias de los profesores de prescolar y primara sobre CTS}

Teniendo en cuenta los instrumentos usados, se procedió a la interpretación de los datos constituidos mediante la triangulación, entendida como una estrategia de análisis cualitativa para saber si aquello que observamos e informamos contiene el mismo significado cuando lo encontramos en otras circunstancias (Stake citado por Perafán, 2004 210). De este modo, primero se sistematizó toda la información obtenida de las observaciones registradas en el diario de campo (observaciones participantes) además de la información proporcionada por las entrevistas semi-estructuradas, en este sentido, se discutieron fragmentos de los datos obtenidos a lo largo de la investigación, en especial lo consignado en el diario de campo.

\section{Creencias sobre la ciencia}

Hablar sobre ciencia y tecnología en un contexto rural implica hacer referencia a las características que definen este escenario, con altos índices de necesidades básicas insatisfechas, y en consecuencia, de su situación, caracterizada por un elevado nivel de pobreza que se manifiesta en condiciones de desempleo y subempleo, deterioro del ingreso y bajo nivel de organización. Tal apreciación se evidencia en las palabras de una profesora de la institución educativa:

"La verdad aquí es difícil hablar de ciencia y tecnología, no contamos con absolutamente nada, teníamos un computador y se lo robaron, aparte, pues imagínese con cinco cursos es bastante complicado y yo sola no puedo hacer mucho, se hacen cositas, como el proyecto de embellecimiento del colegio, o sea sembramos maticas y la idea es poner bonita la escuela".

La idea que la ciencia es una construcción histórica propia de una comunidad, que ha implicado procesos creativos de 
invención y conflictos entre diferentes paradigmas que a su vez representan diversas visiones de mundo, que también encierra intereses y determinadas perspectivas ideológicas, siendo así un conocimiento que no es neutro. Está aún lejos de las creencias que tienen los profesores de las escuelas rurales, y que son similares en otros contextos sociales, una mirada sobre la ciencia que la reduce simplemente a un método científico conformado por un conjunto de etapas que deben ser seguidas rigurosamente.

"La ciencia es método científico, de observación, experimentación, comprobación, análisis y otros elementos que uno debe enseñar a los estudiantes. Pero bueno. Requiere varias cosas, por ejemplo un laboratorio para experimentar. ¿Te has dado cuenta? Yo no he podido hacer ninguna experiencia que propone el libro, pues parte de ello, es la falta de instrumentos y tiempo, aquí en la escuela multigrado toca volar con los conocimientos. Enseñar a cinco cursos al mismo tiempo, para nadie es fácil. Pero todo mundo exige, el rector, la Secretaría de Educación y el Ministerio. Igual ni a los padres les interesa que sus hijos aprendan ciencia, lo que aquí importa es que uno los enseñe a leer y escribir, lo demás es poco importante".

(Palabras de una profesora de la institución).

Lo anterior, no sólo lleva a pensar en la creencia rígida que existe sobre la ciencia sino también como ésta puede orientar las acciones del profesor limitando los procesos de enseñanza al desarrollo de determinadas experiencias de laboratorio. Además, se nota que en este escenario rural, los saberes que deben aprender los niños y las niñas están jerarquizados. Se dedican las primeras horas y la mayor cantidad de tiempo a la enseñanza de la escritura, la lectura y las matemáticas, conservando la idea convencional de que los conocimientos deben ir independientes unos de otros y que aprender ciencias no estaría directamente articulado con el desarrollo de habilidades de lectura y escritura, esto también se evidencia en comentarios de los padres de familia:

"Pues mire profesora, a mí me tocó sacar a los niños de estudiar porque no estaban aprendiendo nada, el Kevin no me sabía ni las vocales, nunca vi que hiciera tareas ni nada, entonces para qué, sólo para que se pongan a jugar será. Aquí hay mucho trabajo y mucha necesidad, además, a los niños les gusta más trabajar que ir a la escuela, se ganan su plática y nos ayudan en las labores de la finca. Si acaso la escuela es para que aprendan a leer, también matemáticas para que no se dejen tumbar en la vida".

(Registro extraído de la conversación con un padre de familia de la institución educativa).

"Los padres de familia exigen cosas útiles para el futuro de sus hijos por ello la lectura, la escritura y la matemática son prioridad, las demás materias son agregados que poco les servirán a los niños".

(Palabras de un docente de grado primero)

También hay otra creencia acerca de la ciencia, que puede ser aproblemática y ahistórica (García et al., 2001), se la considera un conjunto de conocimientos ya elaborados que no requiere de una justificación de los problemas que generaron su construcción, o de cómo ha sido su evolución o qué dificultades ha enfrentado para su desarrollo. 
En las expresiones manifestadas por los docentes se observa una creencia que entiende la ciencia en tanto finalidad, es decir, se concibe como una ciencia inmediata, cuyo objetivo es mejorar y facilitar la vida de las personas y los ambientes de trabajo sin proponer una búsqueda de los sentidos sociales y culturales, sin articularlos a sus aportes y desconociendo que junto a ellos es como se han hecho posibles determinados cambios.

También existe una creencia individualista sobre la ciencia (García et al., 2001), al considerar que los conocimientos científicos aparecen como obra de genios aislados, con esta visión se desconoce el papel del trabajo en equipo. A menudo, la visión individualista se presenta asociada a conceptos elitistas:

"los doctores hacen ciencia", "aquí sólo damos pequeñas bases” , “de pronto en las ciudades los que estudian medicina, tecnologías de alta escala", "aquí los profesores no hacemos ciencia", "los que vienen a revisar las vacas, los cultivos, la flora, son gente de afuera y con mucho estudio, gente con plata que pudo meterse a una universidad".

(Comentarios de los docentes)

El estudio también permite afirmar que los profesores no consideran tan relevante la formación didáctica y pedagógica; saber la materia que se va a enseñar es suficiente para asumir el rol de docente de ciencias. A ello se suma la distancia impuesta por el docente entre los conceptos emitidos en su exposición diaria y la creatividad y recursividad para llevarlos a una acción real que sea más significativa para el estudiante.

Los profesores manifiestan 3 tipos de apreciaciones a la hora de contemplar la posibilidad de hacer ciencia en el contexto rural. La primera se refiere a las oportunidades que brinda el contexto rural para la enseñanza de las ciencias desde una perspectiva contextual, en la que los estudiantes pueden aprender por medio del contacto directo con el ambiente natural. La segunda, denominada "fortalezas", en la cual se instaura la creencia de que en el contexto rural hay herramientas físicas y humanas que permiten aprender ciencia de manera cercana y la tercera, expresa las dificultades para hacer ciencia "avanzada" en el contexto rural.

Se puede ver un contraste entre lo enunciado y la comprensión del término ciencia. Parece que los profesores conciben la oportunidad que les brinda el medio natural para articular acciones o experiencias desde la ciencia, pero no hay coherencia con lo que piensan acerca de la misma, ya que la ven desde el método científico y lejos de sus posibilidades.

\section{Creencias sobre la tecnología}

Relacionado con las creencias que aluden a la tecnología, se encontró que ésta es entendida como una aplicación de la ciencia, como parte del método científico, análoga a los instrumentos que se aplican para un fin, además se entiende lo tecnológico como un resultado de los avances científicos, en tanto que se relaciona con la construcción de máquinas y artefactos.

Este tipo de creencias ya había sido determinada y discutida por Acevedo et al (2005) en otro estudio que muestra la idea limitada de la tecnología vista solo como ciencia aplicada y su identificación con artefactos y máquinas.

Contrario a tal creencia, se habla entonces de tecnología como sistemas y no 
como artefactos inertes. Lo tecnológico no es solo lo que transforma y construye la realidad física, sino también aquello que es capaz de transformar y construir la realidad social de los diversos escenarios humanos.

La creencia de los profesores sobre la tecnología está relacionada con las dificultades para enseñar esta área en las escuelas rurales, e incluye, desde su perspectiva, las deficiencias en su preparación tecnológica. Por otro lado, argumentan que la tecnología en la educación debe ser asumida desde la enseñanza de la informática, el uso de internet y las nuevas técnicas de la información.

Estos resultados coinciden con los elementos determinados por la investigación de Arana et al. (2006, p. 10), que describe una imagen de la "tecnología asociada a aplicaciones del conocimiento científico, fundamentalmente materializadas en aparatos, equipos e instrumentos".

Para las comunidades rurales, las acciones de trabajo y las socializaciones están inmersas en prácticas manuales y artesanales de herencia cultural y procedimental, es decir que hay un tipo de conocimiento desde la cotidianidad, que se basa en la experiencia y en las relaciones con los objetos y los ambientes. Esta práctica es un ejemplo de un tipo de tecnología que valora los procesos de diseño, construcción y evaluación. No obstante, es necesario decir que hay sistemas que, en la construcción social e histórica, han reducido los tiempos y los modos de adquisición y transformación de los productos.

Tal vez por ello, el poblador rural en general, acostumbrado a un sistema manual de trabajo, es resistente a los cambios que trae la tecnología, pues de alguna forma ve remplazada su especificidad y acción por una serie de aparatos de difícil comprensión. A pesar de ello, la tecnología se instaura en la cultura de muchos contextos rurales por sus fuertes repercusiones económicas, además, la tradición histórica de lo rural se ha centrado en la producción. Los campesinos se ven a sí mismos como trabajadores y productores y, por lo general, su vida gira entorno al trabajo desde los primeros años de vida. A continuación se presenta un diálogo con un padre de familia que, para este caso, muestra la emergencia de la tecnología en el contexto rural:

"Qué le comento, aquí en la finca Los Arrayanes la principal actividad es el ordeño. La leche va para Alpina, aproximadamente salen 500 litros diarios, se ordeña en dos jornadas una a las 3: a.m. y la otra a las 2:00 p.m., mis 2 hijos luego de la escuela, por lo general, me acompañan al ordeño, pero esto ha cambiado mucho, antes ordeñábamos manualmente pues el patrón solo tenía 12 vacas y luego el negocio creció y se compró la máquina de ordeño y el tanque de refrigeración que conserva la leche fresca y de calidad hasta que llegue el "carrotanque" de Alpina. Eso aqui nos tocó aprender a manejar la máquina y el computador, antes era un poco más lento todo y ahora con la máquina se sabe la cantidad de leche y calidad que produce cada vaca, es un proceso rápido y lo exige Alpina. Varias fincas de por aquí les ha tocado comprar la máquina para poder vender la leche".

(Registro de un diálogo establecido con un padre de familia).

Es muy importante que la tecnología ocupe cada vez más espacios en el con- 
texto rural, ya que permite el desarrollo procesos educativos que contribuyan a la comprensión de los aspectos culturales y sociales que abarca. Así, no sólo comprendería todos los objetos tangibles utilizados por las personas en su medio, sino también los procesos sociales que la determinan.

Es necesario aclarar que las distinciones hechas entre ciencia y tecnología de ninguna manera pretenden mostrar la ciencia y la tecnología como temas diferentes, por el contrario, estas distinciones se realizaron con el fin de encontrar contrastes en la interpretación de cada una de estas áreas de conocimiento.

\section{Relaciones CTS en conformidad con las creencias determinadas}

Los análisis desarrollados muestran que, en lo que se refiere a las creencias sobre ciencia y tecnología, los profesores de preescolar y primaria, del escenario estudiado, contemplan las relaciones CTS pensándolas desde la aplicación de conocimientos científicos a la invención de artefactos tecnológicos que han de atender una determinada finalidad social. Existe una imagen lineal y funcional de estas relaciones, como aspecto ligado íntimamente a conceptos y materiales sofisticados para facilitar la vida de las personas, que explicaría el reduccionismo de sus creencias acerca de estas interacciones. Tal sistema de creencias representaría una concepción clásica de las relaciones CTS (López y Lujan, 2000).

"Cada vez la tecnología nos atrapa, pues ya es una necesidad tener un celular, es muy chistoso ver a un campesino con su celular de cámara y todo. Hasta los niños tienen celular, mejor dicho, si uno no tiene celular, es un problema para el trabajo e in- cluso para las relaciones emocionales, hasta dependencia crea, si me llamó, que si no me llamó. Mejor dicho el que no esté al día con la tecnología y la ciencia lo aplasta la sociedad".

"La ciencia y la tecnología tienen relación, ya que para estudiar una ciencia se necesita de la tecnología para así comprobar los hechos".

"La ciencia y la tecnología influyen en el ser humano en todo momento, un ejemplo puede ser con los niños de la ciudad y el campo, pues los de la ciudad, tienen un contacto permanente con electrodomésticos y máquinas avanzadas, los del contexto rural no tienen el mismo contacto entonces las relaciones son diferentes".

(Registros diario de campo, relatos de docentes)

Se hace evidente la tendencia a pensar que las interacciones CTS están directamente relacionadas con la aplicabilidad y uso de las tecnologías, o con los beneficios clínicos dados por la ciencia; aunque muchos docentes mencionan en su discurso una influencia de los asuntos de ciencia y tecnología con los problemas reales de la sociedad, no se hace mayor reflexión al interior de las aulas en la comunicación y participación con la comunidad.

"Toda actividad que se realice tiene un fin social. La ciencia busca mejorar la vida y los ambientes humanos, $y$ la tecnología busca facilitar el trabajo de los sujetos".

(Entrevista. Diario de Campo).

"No todos tenemos los recursos económicos para hacer investigaciones científicas, y mucho menos tecnológicas".

(Diario de Campo). 
Las relaciones CTS implican, desde una comprensión amplia e innovadora, la integración de las dimensiones actitudinales, axiológicas y cognitivas de los estudiantes (Martínez y Rojas, 2006). De esta manera, las relaciones CTS no sólo se conciben en la medida de la utilidad o de los beneficios que la ciencia y la tecnología proveen a la sociedad, sino que hacen necesaria la continua reflexión de los profesores junto con los estudiantes, para comprender las problemáticas que apremian en nuestros tiempos a la sociedad. Todo esto se relaciona con lo planteado por López y Valenti citados por Corchuelo y Catebiel (2005, p. 123):

"Aunque el mundo ha sido testigo de una sucesión de desastres relacionados con la ciencia y la tecnología, como vertidos de residuos contaminantes, accidentes nucleares, derramamientos de petróleo, entre otros, estas situaciones permanecen alejadas de las clases. Por tanto, en las aulas se construyen relaciones de tipo triunfalista, resumidas en el llamado "modelo lineal de desarrollo" que prioriza la autonomía de la ciencia básica y el desarrollo sin interferencias de la tecnología para que el crecimiento económico y el progreso social se den por añadidura".

Para profundizar en el análisis desarrollado sobre las creencias de los profesores y las relaciones CTS, también exploramos en los profesores, su metodología de enseñanza, el diseño curricular desarrollado y el uso del libro didáctico.

\section{Metodología de enseñanza y diseño curricular}

La enseñanza que se hizo evidente durante la investigación está basada en la transmisión de contenidos y la utilización del texto como único recurso de enseñanza. Para el área de Ciencias Naturales, la intervención por parte de profesores no se distancia en nada de la comúnmente utilizada para todas las áreas del conocimiento. A pesar de que en el discurso planteado sobre las posibles intervenciones acerca de las relaciones CTS se evoca la realización de experiencias en el ambiente natural de los estudiantes, no se registra ningún tipo de acción diferente de la utilización del texto guía y la secuencia de actividades como dictado, copia, dibujo y solución de talleres de apareamiento: "¿En qué clase estaban? Español. Ah bueno, entonces saquen por favor el cuaderno de ciencias naturales y recordemos. ¿Qué vimos en la clase anterior? El agua. Sí, era el agua, y decíamos que el agua no tiene color, por ejemplo es azul, porque se refleja el cielo. También hablamos del ciclo del agua ¿verdad? Sabemos que el agua se puede encontrar en tres estados: el líquido, sólido y gaseoso. Y ya sabemos por qué ¿cierto? Sí, cuando se pone hielo. Ajá ¿qué estado es? Nadie supo. ¿Sí ven?, es que no estudian. Pues el hielo es estado sólido. Bueno ya pasamos al otro tema, hoy vamos a hablar del suelo. Cerramos la boquita, porque así no puedo dictar y nos demoramos para salir al descanso ¡ustedes verán! A ver, escriban el suelo $y$ debajo pongan esto: el suelo al igual que el aire y el agua, es indispensable para la vida. Sobre el suelo crecen las plantas y viven los animales y las personas.

Hagan el dibujito de lo que acabaron de escribir. ¿Listo? Debajo coloquen: El suelo está constituido por arena, rocas, arcilla, aire y agua. El suelo se 
forma cuando la arena y la arcilla se mezclan con los restos de las plantas y de los animales. ¿Listo? Ahora debajo de lo que escribieron hagan otro dibujito bien bonito y ponemos animales que habiten en el suelo. ¿Ya terminaron? Muy bien, échenle color a esos dibujos, yo les doy tiempo. Los que ya terminaron saquen el libro y resuelvan la página 13 y 14, bueno lo que alcancen a hacer, el resto lo terminan en la casa. En el cuaderno pongan tarea: resolver la pagina $14 y$ la mitad de la 15 del libro, en el cuaderno recortar y pegar animales que vivan en el suelo. Bueno mientras yo empiezo a revisar la tarea de la clase pasada y voy llamando a lista, los demás empiecen a resolver el taller del libro. ¿Y los que no tenemos libro? Pues háganse con un compañero que tenga el libro y hace las actividades. Ya saben de ustedes depende que salgan rápido al descanso".

(Observación clase de ciencias).

El anterior fragmento muestra una metodología de enseñanza caracterizada, primero, por la retroalimentación de la clase pasada, segundo, por la explicación del nuevo tema, acompañado del dictado y el dibujo, tercero, por la utilización del texto guía, cuarto, por la asignación de tarea y quinto, por la revisión de la tarea anterior. La observación se lleva a cabo en una hora de clase, que es el tiempo que se asigna al área dos veces por semana. En términos generales, la metodología de enseñanza de las ciencias es la misma en los 40 registros realizados. La meta, según la docente, es terminar todo el libro, así se enseñarán todos los contenidos propuestos por el currículo de ciencias para el año en curso.
Es importante señalar que en las observaciones realizadas no se desarrolla ninguna experiencia práctica sugerida por el libro y tampoco una acción diseñada por el profesor. Bajo esa metodología de enseñanza hay variadas creencias frente a las relaciones CTS, debido a que las creencias se relacionan con las acciones de los individuos. Con este principio, los profesores en los momentos de enseñanza no dan paso a preguntas de los niños, pareciera que los tiempos destinados para cada clase fueran dominados por el reloj. No se suscitan reflexiones amplias que involucren indagación, confrontación de saberes y experiencias entre los niños y tampoco entre otros agentes de formación.

Tal metodología de enseñanza resulta coherente con lo que plantea Fernández y Eleortegui citados por Triana (2003, p. 106) en su investigación "Qué piensan los maestros acerca de cómo se debe enseñar":

"Profesor transmisor: es aquel para el que la asignatura ya está organizada y su prioridad es el cumplimiento del programa oficial, y deja que los expertos decidan la validez o no de cómo enseñar la asignatura. La clase es únicamente responsabilidad del profesor que ocupa todo el tiempo con su explicación. El libro de texto y unos buenos apuntes, dictados o guiados por el profesor, constituyen toda la documentación que utilizan los alumnos; documentación que es considerada autoridad a toda prueba y que convierte el trabajo del estudiante en un nutrirse de ella y en complementarla con otras informaciones".

Hay elementos que según los profesores inciden considerablemente en el diseño y aplicación de experiencias, por ejemplo, la enseñanza en varios niveles 
de formación que se desarrollen a la vez, y el cumplimiento en la resolución del texto guía para dar cuenta de que todos los contenidos fueron transmitidos a los estudiantes. También es necesario anotar dentro de estos elementos, la asignación de tiempos para cada área y la indisposición de los estudiantes y de la comunidad en general:

"Al hablar de experiencias en ciencias, aquí en el contexto rural, sinceramente se podrían hacer muchísimas por las condiciones del ambiente. Sin embargo, pues aquí no queda tiempo para nada, toca enseñarles a los estudiantes todo lo que trae el libro y no solo eso, hay que cumplirle al rector con una serie de cosas que ni él mismo tiene claro. Según las modificaciones que ha hecho al PEI, tenemos que hacer proyectos productivos, que la granja, que la huerta, en fin, ese señor quiere es mostrar a través de evidencias fotográficas todo lo que se hace en la institución, pero, es que realmente él aspira a un montón de cosas y los profesores no estamos preparados y la comunidad no ayuda para nada. Una vez salimos a hacer una pequeña experiencia fuera del salón. Era mirar una serie de piedras y compararlas, creo que duramos como una hora más o menos fuera del salón. Al otro día recibimos una llamada del rector, pues, según unos padres de familia, habían puesto la queja de que las profesoras y los estudiantes permanecían por mucho tiempo fuera del salón sin hacer nada. Eso me dio mucha rabia, pues, pareciera que nos estuvieran vigilando. La verdad, yo desde ese día prefiero dar la clase normal en el salón y me evito problemas. Además los chicos son súper complicados, no más para una salida, mejor dicho, toca tener los ojos encima de todos, porque son quejas y quejas: me pego, me miro, eso desgasta a cualquiera. Yo me curo en salud y evito todo eso, resuelvo el libro y pues como no hay tiempo para nada más".

(Entrevistas, Diario de campo).

Hay sin duda, una serie de contradicciones institucionales propias de la dinámica escolar, comenzando con el PEI de la institución, que aunque plasma ideales y proyecciones educativas basadas en la comunicación docente, la integración de las sedes, la estrecha relación entre profesores y la puesta en práctica de proyectos productivos que garanticen la formación técnica y científica de los estudiantes desde las realidades propias del campo, no permite, desde la perspectiva docente, la realización de propuestas o estrategias de enseñanza-aprendizaje que se acoplen a los horizontes institucionales. Se encuentran serias dificultades, en la mayoría de los casos, las docentes manifiestan falta de apoyo por parte de la rectoría, que "no ha hecho un recorrido por las sedes para constatar las deficiencias y particularidades de todo lo que debe atender cada profesor", pues no basta con exigir, se necesita una comunicación y apoyo permanente.

Además de lo anterior se nota desmotivación por parte de algunos profesores para trabajar en el contexto rural:

"Venir a esta escuela es todo un sacrificio, yo vivo en Tabio Cundinamarca $y$ debo salir de mi casa mas o menos a las 4 a.m., para estar en Faca a las 6:30 y alcanzar la ruta que me suba a San Rafael, de lo contrario, me tocaría caminar casi dos horas, pues ni un carrito sube. Voy a esperar este año a ver si paso el concurso de la gobernación, porque ahorita estoy en periodo 
de prueba. Pero mejor busco trabajo en Tabio o en la zona urbana de Faca, quien quita de pronto en Bogotá, porque eso de trabajar en el campo y no tener nada de recursos materiales para trabajar con los niños, eso es muy maluco, no tenemos ni siquiera una buena grabadora, mejor dicho nada, eso toca hacer maravillas con lo poquito que se tiene, ahora menos eso de trabajar tecnología, además, sin irnos más lejos, aquí ni los papás le agradecen al profesor lo que se hace por los niños. Aquí los niños son terribles, tienen miles de problemáticas de todo tipo, a mi me ha tocado ser psicóloga, enfermera, y hasta mamá de los estudiantes, pues la familia no colabora para nada".

(Diario de Campo, comentario de una maestra).

Los anteriores aspectos hacen evidente que la comprensión de las relaciones CTS en la enseñanza en estos sectores rurales no puede considerarse al margen de las condiciones y necesidades de formación permanente de los docentes. El estatus profesional y el quehacer educativo han perdido credibilidad social, algunas de las razones involucran la falta de formación continua, falencias investigativas, ausencia de condiciones profesionales favorables apropiadas para el ejercicio docente y una mediana participación de profesores y directivas escolares en la comunidad.

Los profesores perciben las exigencias de los organismos, y de una u otra forma, habría que considerar no sólo las dificultades externas percibidas por ellos, sino también las debilidades profesionales que poseen frente a la planeación, al diseño curricular y en general, a la articulación del conocimiento didáctico del contenido (Mora y Parga,
2007). Puede ser, que no cuenten con autonomía profesional y que carecen de formación para determinar sus propios contenidos y estrategias de enseñanzaaprendizaje:

"Hay quejas por parte de los papás cuando el estudiante al final del año no tiene lleno el libro o el cuaderno de tareas, y se atribuye a la falta de responsabilidad de los profesores porque al parecer no se les está enseñando nada a los estudiantes".

(Diario de campo, comentario de una Docente).

El anterior registro, hace evidente que el docente no tiene autonomía ni estatus profesional en su contexto. Probablemente, porque no ha establecido una comunicación permanente con la comunidad, con los padres de familia, o con sus compañeros de trabajo, para la planeación y reconstrucción de un currículo que valore los diferentes procesos de enseñanza e involucre una evaluación pensada en procesos en lugar de una evaluación centrada en resultados, este ejercicio implica necesariamente la intelectualidad del profesor, no la asistencia reproductiva de conocimientos construidos por otros.

En ese contexto, Mora y Parga (2007, p. 101) destacan la ausencia del diseño curricular por parte de los docentes:

"La profesionalización del ejercicio docente es un proceso inseparable de su objeto epistemológico, el diseño curricular y su investigación Pedagógico -didáctica en los procesos de enseñanza- aprendizaje en el aula. En este sentido, el ejercicio docente se sustenta únicamente en los conocimientos generales logrados durante la formación, muchas veces terminal, obtenida en las facultades de educación; tampoco se circunscribe en la 
acción técnica de enseñar contenidos elaborados por expertos; de igual manera no es una manifestación espontaneista, individual y artesanal de cada docente como consecuencia de su conocimiento experiencial y empírico logrado a lo largo de su experiencia docente en las aulas de clase; por el contrario, es una integración de trabajo profesional de coparticipación colectiva y permanente de grupos de docentes en sus contextos escolares en torno a la reflexión sobre el qué y el cómo enseñar".

Por otro lado, el contexto rural reúne tal vez, una serie de elementos que son resistentes al cambio educativo, lógicamente esto depende en buena medida, del profesionalismo de los docentes, las creencias que se tengan las relaciones CTS en el ambiente escolar y su correspondiente abordaje en los procesos de enseñanza-aprendizaje de las ciencias naturales. En tal sentido, aunque los profesores justifican su falta iniciativa frente al diseño curricular y las planeaciones personales en determinados problemas estructurales, como la extensión de los contenidos de las descripciones curriculares oficiales y la falta de tiempo, de acuerdo con Acevedo (1996) estas actitudes también implican problemas relacionados con sus creencias, tales como los siguientes:

- Una formación que es básicamente disciplinar para abordar algo que es esencialmente multidisciplinar.

- Un cierto temor a perder su identidad profesional, lo que en parte está relacionado con la percepción que tienen de las finalidades de la enseñanza de las ciencias.

- Sus creencias sobre la naturaleza de la ciencia, tanto en los aspectos epistemológicos como en los sociológicos.
- El carácter más abierto, dialéctico y provisional de los materiales curriculares CTS y la propia evaluación de las cuestiones CTS, lo que da lugar a cierta inseguridad por parte del profesorado.

- La poca familiaridad de la mayor parte del profesorado con las estrategias de enseñanza-aprendizaje y con los criterios, normas, técnicas e instrumentos de evaluación que se requieren en la enseñanza CTS.

. El que las estrategias adecuadas y estimulantes, para la mayoría del alumnado, pudieran resultar demasiado exigentes para muchos profesores.

- Resistencias comunes a todas las innovaciones debido al carácter generalmente conservador de los sistemas educativos.

Hablar de autonomía y diseño curricular en las zonas rurales depende en buena parte de la formación profesional del docente y de su capacidad para asumir modalidades del ambiente educativo en escuelas rurales (multigrado, aceleración del aprendizaje, escuela nueva, propuesta PER), es decir, muchos profesores tienen falencias en su formación inicial y no cuentan con formación permanente en las áreas de conocimiento que tienen la responsabilidad de orientar:

"Sinceramente yo no fui formada profesionalmente para enseñar ciencias naturales, soy licenciada en preescolar, pero al iniciar el año se organizaron los docentes rotativos para la sede San Rafael. Cuando me dijeron que debía escoger un área para dictar, no me sentí preparada para escoger matemáticas porque aunque soy buena para ello, los niños tienen muchos conflictos con esa asignatura; tampoco escogí artes pues soy malísima para todo eso; y educación física tampoco me gusta. Por eso asumí la enseñanza de las ciencias 
naturales, ética y religión. Ciencias porque me llama la atención y me gusta la naturaleza. Ética pues es importante formar valores así como la religión".

(Diario de campo).

El testimonio presentado lleva a pensar que la acción pedagógica está motivada por los intereses y gustos del profesor, lo cual es importante, pero además, requiere de una formación permanente y multidisciplinar que implique aprendizajes recíprocos con sus estudiantes y colegas.

\section{Uso del libro didáctico}

Sin duda, uno de los elementos que guía el accionar pedagógico y didáctico de los docentes en escenarios rurales lo constituye el libro didáctico seleccionado y aprobado institucionalmente al iniciar el año escolar:

"Yo llegué nueva este año al Policarpa

Salavarrieta. Cuando empezamos el año escolar obviamente debíamos acomodarnos a las propuestas del rectory de los docentes antiguos. Yo traía otra metodología donde no necesariamente debía ajustarme a un único libro, sino a varios. En el primer mes del año, lo que uno hace es reunirse con los profesores y allí nos visitan varias editoriales, se revisan los libros y también se acogen las sugerencias y publicidad que trae cada representante de la editorial. "Por consenso", pero más por decisión del señor rector se escogió el libro integrado de Santillana. Es muy completo, pues vienen 2 libros integrados para los estudiantes para cada nivel, uno integra ciencias naturales, ciencias sociales y matemáticas. El otro integra español, inglés y comprensión lectora. Para los profesores trae una guía completa que integra los dos libros. También trae una cartilla de estándares, indicadores de logro, competencias y formatos evaluadores. Eso a todos los profesores nos pareció muy bueno, pues por ejemplo los que estamos en la sede Multigrado o bi-docente, es un poco más fácil con estos libros. Igual la editorial hace unos descuentos cuando el colegio hace contrato con ellos, para que todos los estudiantes adquieran ese libro. Aquí la mayoría de los estudiantes ya lo compraron. Para los de preescolar, no se les pide libro Santillana, a ellos se les solicita otro tipo de texto según su edad, por lo general, se les pide libro de ediciones creativas. No es muy completo pero igual uno nunca va a encontrar un libro completo para los niveles de preescolar".

(Diario de campo, conversación con una profesora).

La presencia de actores extraescolares, como las editoriales, juega un papel decisivo en el uso del libro didáctico por parte de los profesores, no sólo en los contextos rurales sino también en los urbanos, tradicionalmente, el trabajo de las editoriales consiste, al iniciar el año, en persuadir y convencer a las instituciones escolares para que adquieran sus textos guía, por lo que su presencia tiene un carácter comercial e indiscutiblemente económico.

A pesar de los problemas en el libro didáctico, los profesores lo usan frecuentemente y, en el caso de los profesores participantes de este trabajo, se observó en múltiples episodios que el abordaje de contenidos, en los libros susceptibles de articular relaciones CTS, se limita a mostrar los usos de la ciencia y la tecnología en la vida cotidiana, confirmando la creencia acerca del carácter utilitario de la ciencia y la tecnología, 
dejando de lado la reflexión social, es más, se desconocen las realidades que envuelven el contexto de los estudiantes, en este caso del escenario rural. Frente a este problema es pertinente resaltar las contribuciones de García et al. (2001, p. 149):

La educación CTS no solo comprende los aspectos organizativos y de contenido curricular, debe alcanzar también los aspectos propios de la didáctica. Para empezar, es importante entender que el objetivo general del docente es la promoción de una actitud creativa, critica e ilustrada, en la perspectiva de construir colectivamente la clase y en general los espacios de aprendizaje. En dicha "construcción colectiva" se trata, más que de manejar información, de articular conocimientos, argumentos y contra argumentos, sobre la base de problemas compartidos, en este caso relacionados con las implicaciones del desarrollo científicotecnológico.

Es muy probable que las creencias sobre ciencia y tecnología impidan a los profesores participantes del estudio establecer relaciones críticas entre CTS, ya que éstas son percibidas desde un punto de vista utilitario y reducido a meras necesidades. Es evidente la tendencia a pensar que ciencia y tecnología están directamente relacionadas con la aplicabilidad y utilidad social, sin embargo, no se hace mayor reflexión al interior de las aulas sobre sus repercusiones e impactos sobre la calidad de vida de los ciudadanos.

Pareciera ser que las relaciones están ligadas exclusivamente al uso de la ciencia y a las funciones de los artefactos (funcionamiento, manipulación y control). Así, la participación democrática en asuntos científicos y tecnológicos no es considerada.

Las relaciones CTS desde una comprensión amplia implican la definición de nuevos roles docentes y una redefinición de la interacción con los estudiantes, no solo se conciben en la medida de la utilidad o los beneficios que puedan traer a la sociedad (Martínez y Rojas, 2006).

\section{Conclusiones}

A manera de conclusión, en la figura 1 mostramos una síntesis de los aspectos relacionados con las creencias de los profesores sobre CTS, buscando entenderlas como parte de la compleja dinámica escolar que abarca orientaciones institucionales, elementos del contexto social y la acción del profesorado expresada en la metodología de enseñanza utilizada. Es importante comprender estas interacciones para adelantar procesos de formación permanente, con los profesores del contexto rural, que contribuyan en reflexionar sobre la dinámica de la ciencia y la tecnología en el mundo contemporáneo con miras a transformar las prácticas docentes.

En general, los profesores que participaron en el estudio realizado, presentan una creencia rígida sobre la ciencia que se reduce al desarrollo de un método, así, los procesos de enseñanza se limitan a la transmisión de los pasos correspondientes de este método, unido a esto, los docentes piensan que no hay relación entre los contenidos científicos escolares y el trabajo de lectura y escritura que se prioriza en los estudiantes de preescolar y básica primaria.

Otra creencia se asocia a una visión de la ciencia como aproblemática, ahis- 
tórica e individualista, considerándola como un conjunto de conocimientos creados por personas aisladas de la dinámica social, que a su vez, requieren de una transmisión fidedigna que desconoce el qué y el cómo ha cambiado el conocimiento de la ciencia que se enseña (Mora y Parga, 2008).

En cuanto a la tecnología, se cree que ésta es una mera aplicación de conocimientos científicos o que representa la construcción de artefactos y máquinas.

Aunque tales creencias limitan el establecimiento de relaciones entre CTS al interior de la escuela, este panorama no se debe exclusivamente a los profesores, ya que la creencia es una construcción social y cultural articulada a las condiciones de profesionalidad del docente y a las características del contexto rural, por tanto, tales creencias no significan de modo alguno que los profesores no deseen participar de una renovación curricular de tipo CTS o que el contexto rural no brinde posibilidades para eso, de lo que se trata es de promover procesos de formación del profesor que valoren la realidad del educador rural que, en muchas ocasiones, se encuentra distante de procesos de cualificación que le posibiliten discutir sobre las implicaciones sociales de la ciencia y la tecnología y su respectiva relación con procesos de enseñanza-aprendizaje. De esta forma, se podría favorecer la construcción de currículos flexibles y contextualizados diseñados por el propio profesorado.

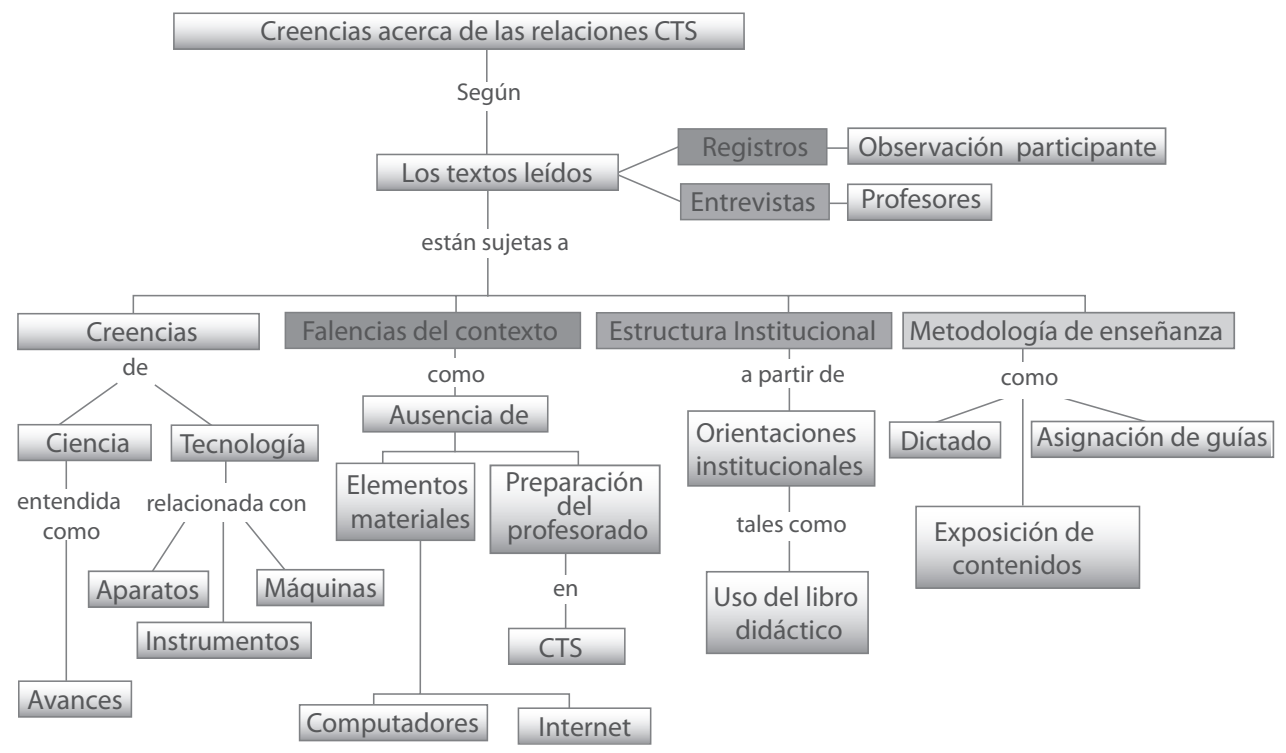

Figura 1. Creencias de los profesores sobre CTS en el escenario de la escuela rural. 


\section{Referencias bibliográficas}

Acevedo, J.; Vázquez, A., Mannasero, M. y Acevedo, P. (2005). Aplicación de una nueva metodología para evaluar las creencias del profesorado sobre tecnología y su relación con la ciencia. Educación Química, 16(3), 372-382.

Acevedo, J.; Vásquez, A., Mannassero, M. y Acevedo, P. (2002a). Actitudes y creencias CTS de los alumnos: su evaluación con el cuestionario COCTS, obtenido el día 12 de Febrero de 2006, desde http://www.oei.es/revistactsi/ numero2/varios1.htm

Acevedo, J.; Vázquez, A., Mannasero, M. y Acevedo, P. (2002b). Persistencia de las actitudes y creencias CTS en la profesión docente, Revista Electrónica de Enseñanza de las Ciencias. 1(1), obtenido el 12 de Febrero de 2006, desde http://www.saum.uvigo.es/ reec/volumenes/volumen1/Numero1/Art1.pdf

Acevedo, J.; Vázquez, A. y Manassero, M. (2002). Evaluación de actitudes y creencias CTS: diferencias entre alumnos y profesores, Revista de Educación, 328, 355-382, obtenido el 15 de Junio de 2006, desde http:// www.campus-oei.org/salactsi/acevedo14.htm

Acevedo, J. (2000). Algunas creencias sobre el conocimiento científico de los profesores de educación secundaria en formación inicial, Bordón, 52(1), 5-16, obtenido el 25 de Junio de 2006, desde http://www.oei.es/salactsi/ acevedo18.htm

Acevedo, J. (1996). La formación del profesorado de enseñanza secundaria y la educación CTS. Una cuestión problemática, Revista Interuniversitaria de Formación del Profesorado, 26, 131-144, obtenido el 15 de Junio de
2006, desde http://www.campus-oei. org/salactsi/acevedo9.htm

Ander Egg, E. (1978). Introducción a las técnicas de investigación social. Argentina: Magisterio.

Álvarez-Gayou, J. (2004). Cómo hacer investigación cualitativa. México: Paidós.

Arana, M.; Sierra, L., Sequeda, S., Rodríguez, Y., Patarroyo, G., Ruíz, D., Agudelo, R. y Merchán, O. (2006). Informe Final de Investigación del proyecto DSISI-017-05 "Estrategias de educación científico y tecnológicas para el proceso de formación profesional de educadores infantiles en las universidades Pedagógica Nacional y Libre de Colombia”. Bogotá: CIUP.

COLCIENCIAS. (2005). La percepción que tienen los colombianos sobre la ciencia y la tecnología, Bogotá: COLCIENCIAS.

Corchuelo, M. y Catebiel, V. (2005). Orientaciones Curriculares con el enfoque CTS para la educación media. Tecné, Episteme y Didaxis. 18, 121-131.

Del Rincón, D.; Arnal, J., Latorre, A. y Sans, A. (1995). Técnicas de investigación en Ciencias Sociales, Madrid: Dykinson, 263-303.

Gallego, R.; Torres, L. y Aristizábal, A. (2004). Análisis epistemológico y didáctico de los estándares en Ciencias Naturales, Educación y Cultura, 66, 36-40.

García, E.; González, J., López, J., Lujan, J., Gordillo, M., Osorio, C. y Valdés, Célida (2001). Ciencia, tecnología y sociedad: una aproximación conceptual, España: OEI.

Guerrero, J. y Cabrera, L. (2004). Estrategia didáctica para la enseñanza de la Química en el contexto de la relaciones ciencia, tecnología, sociedad y 
ambiente.Tecné, Episteme y Didaxis, 16, 132-145.

Guerrero, J. y Cabrera, L. (2002). Enseñanza de las Ciencias Experimentales, mediante enfoque interdisciplinar utilizando el impacto ambiental generado por emisiones de $\mathrm{CO}_{2(\mathrm{~g})}$. Tecné, Episteme y Didaxis, 12, 72-81.

Guerrero, J. (2000). Impacto ambiental generado de la actividades químicas de producción y uso de fluorocarbonos. Tecné, Episteme y Didaxis, 7.

López, J. y Lujan, J. (2000). Ciencia y política del riesgo. Madrid: Alianza Editorial.

Martínez, E. y Reyes, S. (2006). Estado de desarrollo de las relaciones CTS en el colegio departamental Tapias, municipio Chipaque, Trabajo de Grado, Bogota: UPN.

Martínez, L. y Rojas, A. (2006). Estrategia didáctica con enfoque ciencia, tecnología sociedad y ambiente, para la enseñanza de tópicos de bioquímica, Tecné, Episteme y Didaxis, 19, 44-62.

Martínez, L.; Peña, D. y Villamil, Y. (2007). Relaciones ciencia, tecnología, sociedad y ambiente, a partir de casos simulados: una experiencia en la enseñanza de la química.Ciência \& Ensino, 1 (número especial), obtenido en enero de 2008, desde http://www.ige.unicamp.br/ojs/ index.php/cienciaeensino/article/ viewFile/145/111.

Mora, W. y Parga, D. (2007). Tramas histórico-epistemológicas en la evaluación de la teoría estructural en química orgánica,Tecné, Episteme y Didaxis, $21,100-118$.

Parga, D. (2007). Diez años de investigación didáctica en el departamento de Química,Tecné, Episteme y Didaxis, 22, 171-183.

Mora, W. y Parga, D. (2008). El conocimiento didáctico del contenido en química: integración de las tramas de contenido histórico-epistemológicas con las tramas de contexto/aprendizaje, Tecné, Episteme y Didaxis, 21, 100-118.

Perafán, A. (2004). La epistemología del profesor sobre su propio conocimiento. Bogota: UPN.

Reyes, L.; Salcedo, L. y Perafán, G. (1999). Acciones y creencias: Tesoro oculto del educador, Tomo I. Bogotá: Universidad Pedagógica Nacional.

Triana, A. (2003). Sentido y cultura de la escuela rural. Tunja: Colciencias.

Vásquez, A. y Manassero. (2005). Proyecto de investigación iberoamericano en la evaluación de actitudes y creencias relacionadas con la ciencia, la tecnología y la sociedad. En Memorias I Congreso Iberoamericano de CTS +1 . México: OEI. 